\title{
Defining Effective Gain for Evaluation of Orbital Angular Momentum Links
}

\author{
Elaheh Shamoushaki*, Hadi Aliakbarian \\ Department of Electrical Engineering, K. N. Toosi University of Technology, Tehran, Iran \\ Received 26 December 2020; received in revised form 14 February 2021; accepted 21 February 2021 \\ DOI: https://doi.org/10.46604/aiti.2021.6922
}

\begin{abstract}
In this paper, a communication link based on circular phased array antennas generating orbital angular momentum (OAM) beams at radio frequency is investigated. The presence of a null in the radiation pattern of OAM antennas is the main drawback of them. This problem makes it difficult to establish a telecommunication link using OAM systems and calculate the link budget for such a system. To solve this problem, we have defined two new gain parameters by using Friis Transmission Equation. The new formulas can help to calculate the effective gain of OAM antennas. Also, we have defined the effective OAM gain in detail for the first time in order to evaluate the performance of the OAM links. By using the proposed formulas, a capable and secure link based on the orthogonality of OAM beams can be designed.
\end{abstract}

Keywords: circular array antenna, effective OAM gain, helical phase front, orbital angular momentum, OAM link

\section{Introduction}

Due to the significant progression of communication systems in recent centuries, the efficiency and security of such systems have been considered as essential issues [1]. Orthogonality of electromagnetic waves is a property of them that can be used to transmit different information on the same frequency in a communication system. This property causes the channel capacity to increase and also makes the channel more secure [2-5]. Orbital angular momentum (OAM) is a way that has been introduced to realize this property of electromagnetic waves.

During the last two decades, OAM of light has been studied in optics with Laguerre-Gaussian beams [6]. Recent reports have shown that OAM can be used in the radio frequency both theoretically [7-8], and experimentally [3]. Researchers have presented different techniques to generate OAM carrying beams at optical frequency ranges or RF ranges. At optical frequency, these techniques contain plasmonic meta-surfaces [9], holograms [10], liquid crystal-based spatial light modulators [11] and spiral phase plates [7]. At RF frequency the presented techniques are circular array antennas [4, 8, 12], spiral phase plates (SPP) [1], twisted parabolic reflectors [3] and transmit arrays [13]. The first simulation of OAM in radio frequency was performed in 2007 [14]. Spiral phase plate has a simple structure, but when multiple SPPs are used in an OAM carrying beam, multiplexing and de-multiplexing cannot be implemented easily [14]. Circular array antenna is the typical structure to radiate different OAM beams simultaneously.

One of the key problems of OAM systems is the presence of a null in the middle of their radiation pattern. This problem is discussed in the present paper. As a result, not only it is difficult to establish a telecommunication link using OAM systems, but also, we are not really sure about how to calculate the link budget for such a system as theoretically the antenna's gain is zero in its main direction. Therefore, the main problem which is considered here is to define a value in which one can use in its calculations, called effective OAM gain.

* Corresponding author. E-mail address: elaheshamushaki@email.kntu.ac.ir 
The structure that was used in this paper, similar to $[4,8]$, is a phased circular array with each element having progressive $2 \pi l / N$ phase difference, which leads to different orbital angular momentum (OAM) mode numbers $(l)$. The proposed structure is designed, simulated, and then used in a communication link to analyze the rate of receiving information in such systems by varying the distance between the transmitter and the receiver. It should be emphasized that for generating electromagnetic waves carrying OAM beams, it is not necessary to use circular phased array antennas, but it is sufficient [8]. Also, an effective gain is defined and introduced in order to evaluate OAM links.

\section{Theoretical Background}

When a wave beam has an amplitude distribution of $u(r, \varphi, z)=u_{0}(r, z) e^{i l \varphi}$ in the transverse plane, it carries angular momentum over the beam axis over a free space volume $V$, which is defined as follows.

$$
J=\int \varepsilon_{0} r \times \operatorname{Re}\left\{E \times B^{*}\right\} d V
$$

where $\varepsilon_{0}$ is the free space permittivity and $*$ denotes a complex conjugate.

This angular momentum is decomposed into Spin Angular Momentum (SAM) and Orbital Angular Momentum (OAM). Spin angular momentum is related to the circular polarization of the wave beam, where orbital angular momentum is not related to the polarization of the light beam, and it is an extrinsic rotation of the electromagnetic wave. In fact, OAM is related to the spatial structure of the wave-front [15]. Decomposition of angular momentum into SAM and OAM is known as Humblet decomposition, i.e.,

$$
J=L+S
$$

where

$$
\begin{aligned}
& L=\varepsilon_{0} \int \operatorname{Re}\left\{i E^{*}(\widehat{L} \cdot A)\right\} d V \\
& S=\varepsilon_{0} \int \operatorname{Re}\left\{E^{*} \times A\right\} d V
\end{aligned}
$$

The OAM operator is occurred as $\hat{L}=-i(r \times \nabla)$ in $(3)[4,8,16]$. OAM modes with unlimited eigenstates can improve the capacity of the communication system, comparing with SAM with only two orthogonal states.

The most usual antenna structure to generate OAM beams is uniform circular (ring) array, similar to the structure of Fig. 1(a). In general, when the goal is directivity, the whole circular plane is filled with antenna elements, which is called circular array in contrast with ring array in OAM having elements only on its circumference. The fundamental theory of circular ring arrays has been studied in [17]. Such a structure of Fig. 1(a) is normally used for end-fire radiation pattern in the horizontal plane with elements pointing in different directions. In contrast, in OAM applications, the radiation is desired in the broadside direction with the element pointing in the same direction, namely broadside. For such an array, one can formulate the radiation pattern of the array as the product of the element factor and the array factor, in which the array factor is given by the following equation:

$$
A F=\sum_{i=1}^{N} \exp \left[j k a \sin \theta \cos \left(i \frac{2 \pi}{N}-\phi\right)\right]
$$

where $N$ is the number of elements in the array, $k=2 \pi / \lambda$ and $a$ is the radius of the ring. This radiation pattern is very similar to the $0^{\text {th }}$ order of Bessel function of the first kind $\left(J_{0}\right)$, when the number of elements approaches infinity [18]. 


\section{Null of a Circular Array Antenna}

Using a circular phased array antenna is a way to generate electromagnetic waves carrying OAM beams. Such antennas can generate several OAM modes just by tuning the feeding network or even having multiple beams feeding network to excite several OAM modes simultaneously. Assigning different information to each of these OAM modes can increase the capacity of a communication channel.

In this section, a circular phased array antenna that generates electromagnetic wave carrying OAM is simulated and later used for our study. In the proposed antenna, $N$ array elements are distributed equidistantly along a circle's perimeter. The radiating elements have identical amplitudes and they all point in the same direction. Two adjacent elements have the phase difference $2 \pi l / N$, and the $n^{\text {th }}$ element has the phase $\phi_{n}=l \varphi_{n}$ where $l$ determines the OAM mode number. Possible values of OAM modes are limited due to the number of array elements, where OAM mode numbers greater than $l=N / 2$ will not generate a pure rotating phase front [8]. The characteristic of an OAM beam is its helical phase front. The magnitude and the sign of $l$ defines the number of intertwined helices and the handedness, respectively [6].

In the proposed structure, 12 dipole elements with the length of $\lambda / 2$ are placed in $x-y$ plane, in $y$ direction, operating at the frequency of $2.4 G H_{Z}$. The elements are phased such that two adjacent elements have the phase difference $2 \pi l / N$.

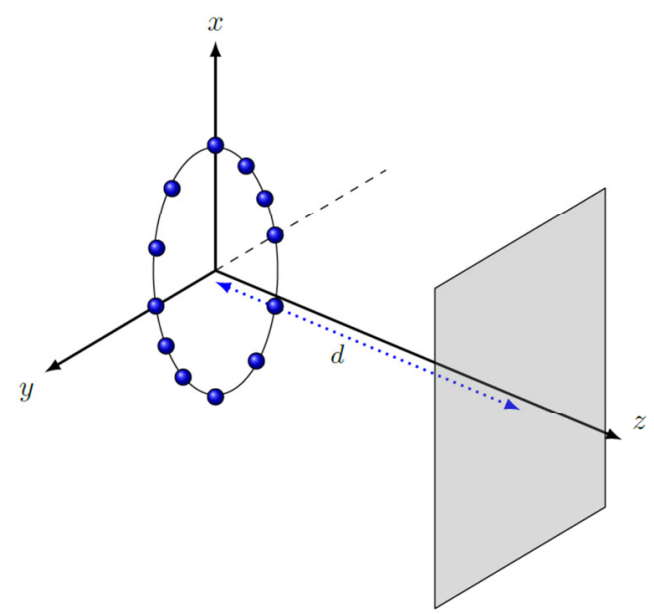

(a) Structure of a circular array antenna and a monitoring screen

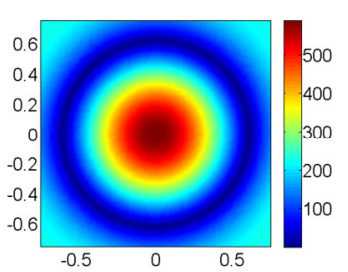

(b) $l=0$
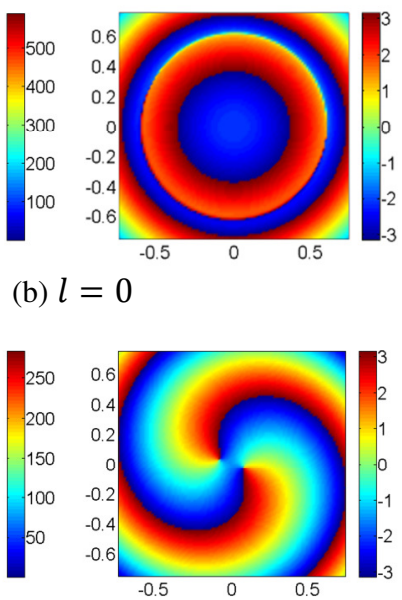

(d) $l=2$
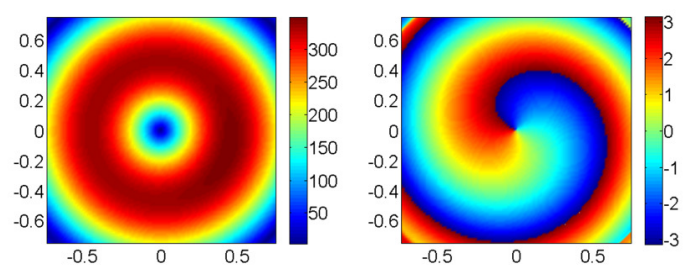

(c) $l=1$
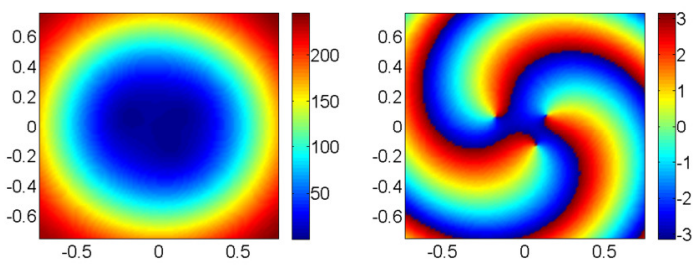

(e) $l=3$

Fig. 1 Different OAM mode numbers of a circular array antenna

Fig. 1(a) shows the schematic of a circular array antenna with 12 dipole elements along the perimeter of a circle with the array diameter of $4 \lambda$ operating at the frequency of $f=2.4 G H_{Z}$ and a monitoring screen away from it at the distance of $25 \lambda$ to show the electric field of different OAM mode numbers. The amplitude (left) and the phase (right) of the electric fields for 
different OAM mode numbers at $d=25 \lambda$ meters distance from the antenna are simulated using MATLAB, as shown in Fig. 1(b)-(e). According to diffraction theory, when OAM beams propagate in free space, they diverge. By increasing the mode number of OAM beams, they diverge faster, and consequently, the width and the depth of the null increases, as is shown in Fig. 1(b)-(e).

The schematic of the proposed circular array antenna simulated using CST is depicted in Fig. 2, which has 12 dipole elements located on a circle's perimeter and operating at the frequency of $f=2.4 G H_{Z}$. The diameter of the array is $2 \lambda$. The length and the radius of the dipoles are $0.5 \lambda$ and $0.001 \lambda$, respectively.

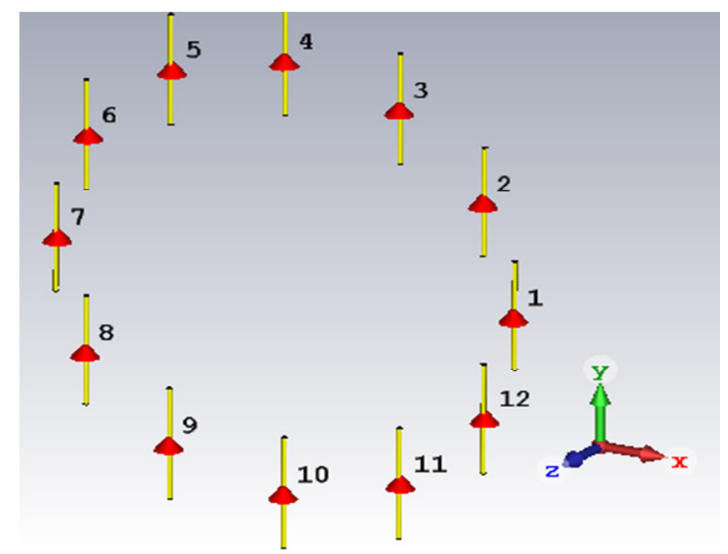

Fig. 2 Schematic of the circular array antenna

The reflection magnitude vs frequency plot for the proposed phased array antenna generating OAM mode number of $l=0$ is presented in Fig. 3 .

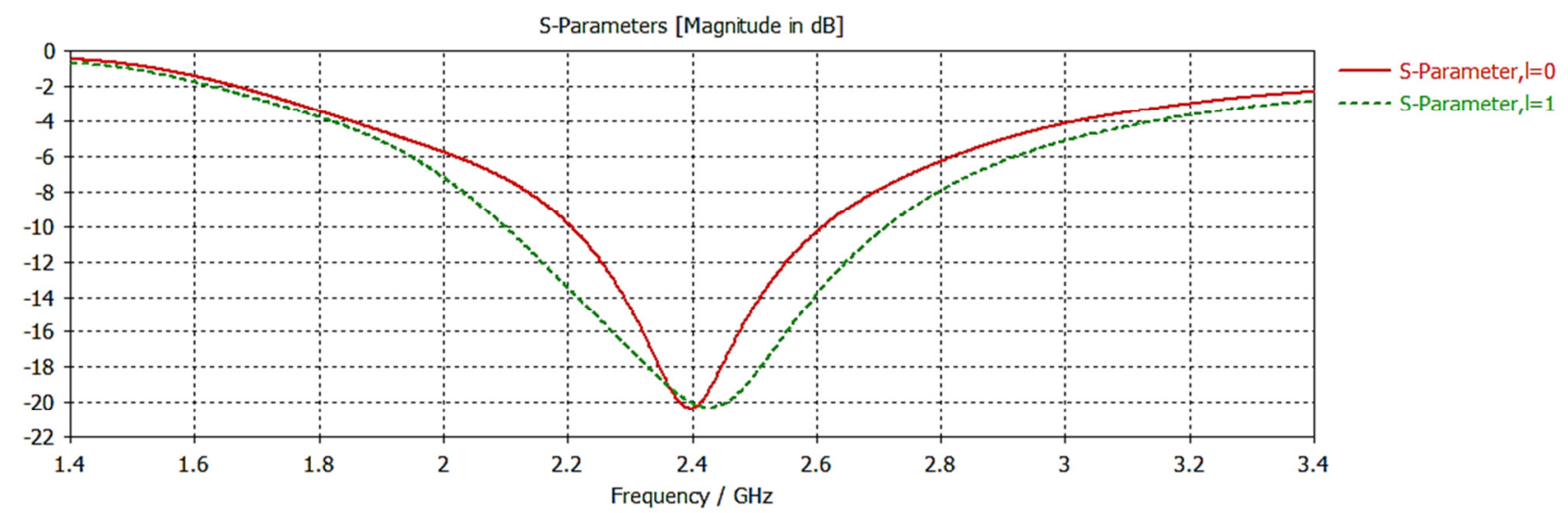

Fig. 3 Diagram of the reflection magnitude

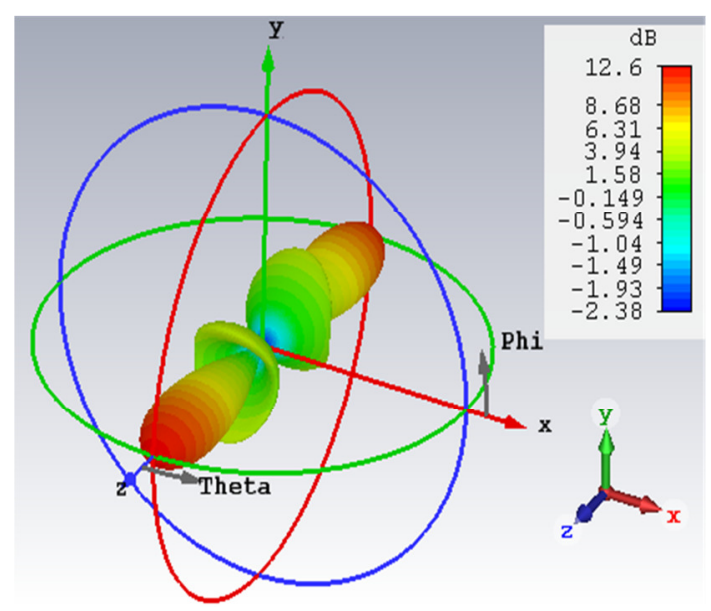

(a) $3 \mathrm{D}$ radiation pattern

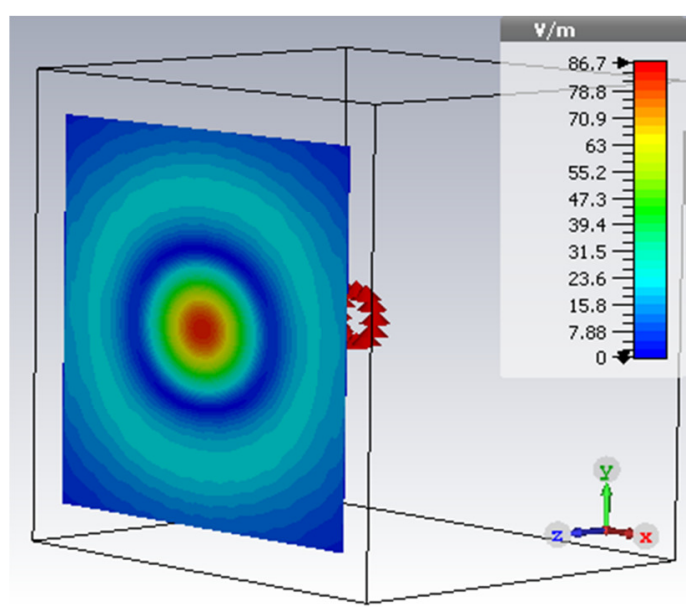

(b) Phase front

Fig. 4 Simulated results of the circular array antenna with OAM mode number of $l=0$ 
For an antenna carrying OAM mode number of $l=0$, the phase difference between adjacent elements is $0^{\circ}$, thus the wave front is like a plane wave. The $3 \mathrm{D}$ radiation pattern and the phase front of the circular array antenna carrying OAM mode number of $l=0$ is shown in Fig. 4. As shown in Fig. 4(a), the beam maximum is in the direction along the axis of the array and there is no null in this direction of the pattern.

In the proposed antenna with OAM mode number of $l=1$, the phase difference between two adjacent elements is $2 \pi l / N=30^{\circ}$. For this purpose, a controlled phase shift feeding network has been used. Using this phase difference, causes the circular array antenna to generate beams carrying OAM mode number of $l=1$. The reflection magnitude versus frequency plot for the proposed antenna is presented in Fig. 3.

The 3D radiation pattern and the phase front of such antenna are shown in Fig. 5. As shown in Fig. 5(a), a null is created along the axis of the array, and the main lobe magnitude is weaker in comparison to Fig. 4(a). In such an antenna, as shown in Fig. 5(b), the phase front is helical.

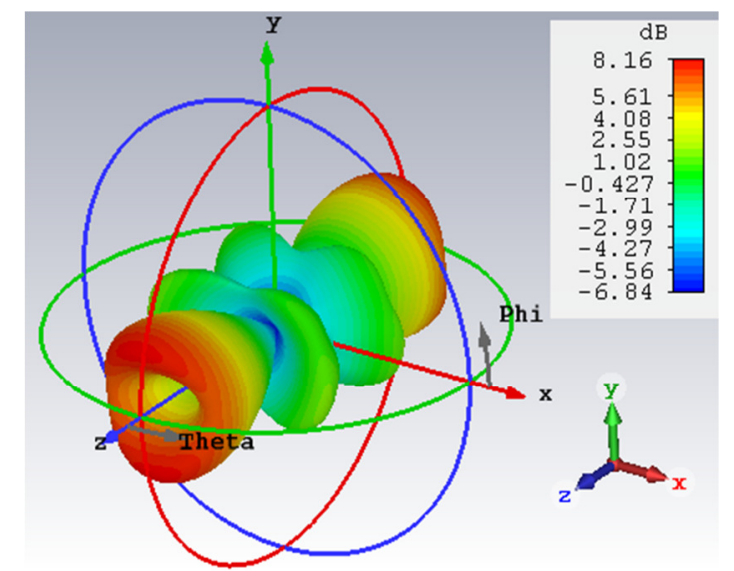

(a) $3 \mathrm{D}$ radiation pattern

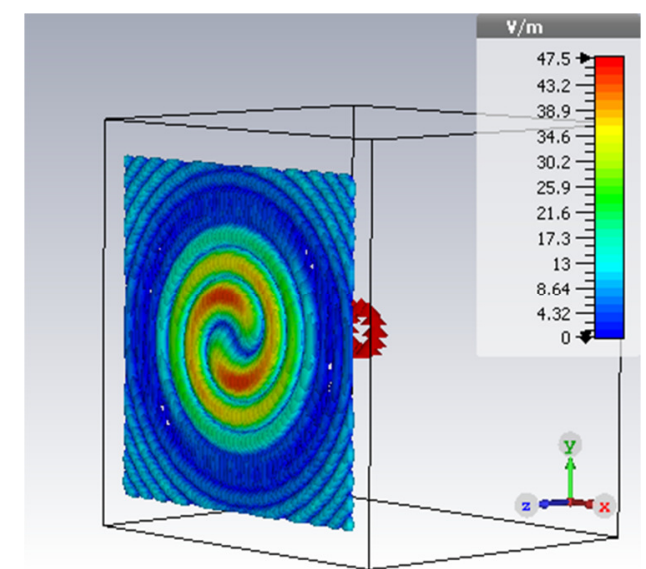

(b) Phase front

Fig. 5 Simulated results of the circular array antenna with OAM mode number of $l=1$

\section{Null Performance in an OAM Link}

For an aperture antenna, the far field is proportional to the Fourier transform of the aperture field [19]. To understand the far field behaviour of an OAM antenna, a communication link is proposed by using a transmitter located on the left side and a receiver located on its right side, in the broadside direction of the transmitting antenna. The transmitter and receiver are circular array antennas with 12 dipole elements along the perimeter of a circle with the array diameter of $2 \lambda$, as shown in Fig. 6(a).

In the proposed structure, $\rho^{\prime}$ and $\phi^{\prime}$ denote the radial and angular variables, the aperture field which is denoted by $f$ and $k^{\prime}$ shows the radial coordinate in the spectral domain, therefore, according to [20], the far field power pattern of the source is given by the following equation in (6),

$$
F_{l}\left(k^{\prime}\right)=\int_{0}^{\infty} f_{l}\left(\rho^{\prime}\right) J_{l}\left(k^{\prime} \rho^{\prime}\right) \rho^{\prime} d \rho^{\prime}=H_{l}\left\{f_{l}\left(\rho^{\prime}\right)\right\}
$$

which is the Hankel transform of order $l$ of the function $f_{l}\left(\rho^{\prime}\right)$ and $J_{l}$ is the $l^{\text {th }}$ order Bessel function of the first kind.

According to (6), for $l \neq 0$ the value of $J_{l}(0)$ is zero, which means the presence of a null in the boresight direction of the source. Due to the characteristics of Bessel function, increasing the number of $l$ causes the size and the depth of the null to increase. Therefore, when a receiver is located in the broadside direction of the source, increasing the number of $l$ makes the receiving signal even weaker. This causes the transmitted signal to be wasted. Also, increasing the distance of the receiving antenna from the transmitter causes an increase in the depth and the size of the null. 
In this section, OAM antennas are used in a communication link in order to improve the capacity and the security of the channel. Therefore, the null performance and the rate of receiving the information have been analyzed as well. For this purpose, receiving and transmitting information in a communication link, and using two circular array antennas with 12 dipole elements along the perimeter of a circle with array diameter of $2 \lambda$ is considered, operating at the frequency of $2.4 G H_{Z}$. The simulated structure using CST is shown in Fig. 6(b).

In the following, three different feeding networks of the circular array antennas providing $l=0$ and $l=1$ OAM modes and their effects on a communication link are considered using CST.

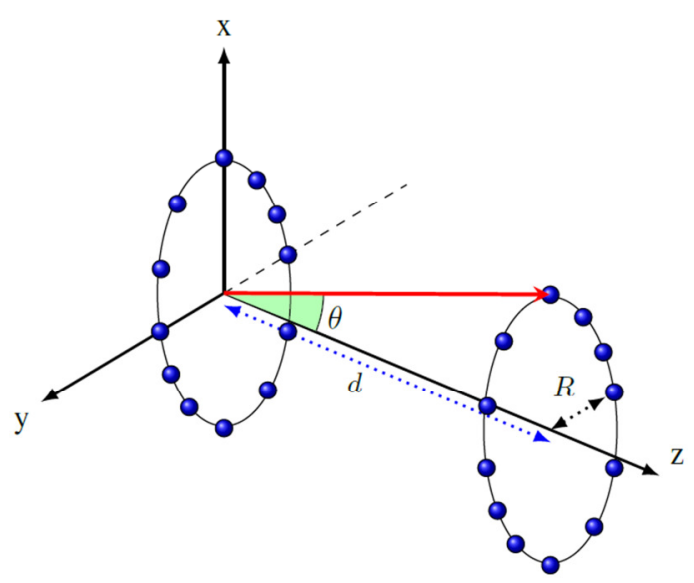

(a) Schematic of the communication link

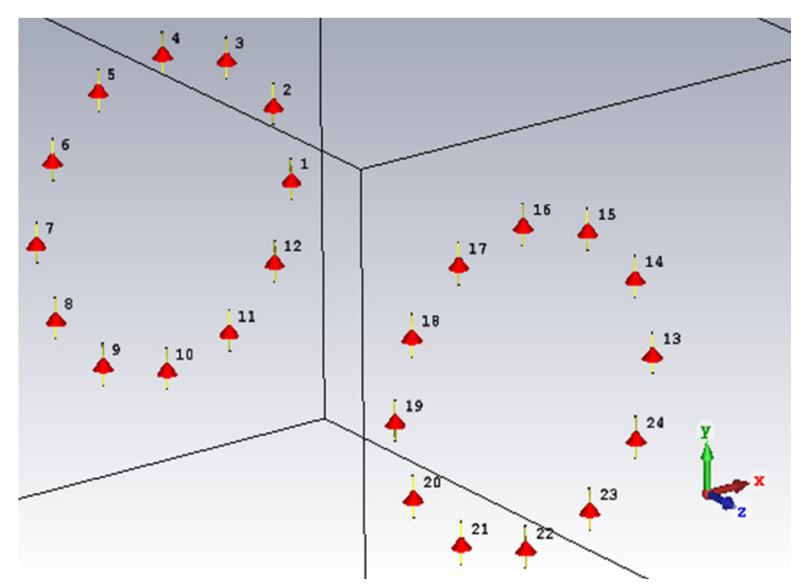

(b) The simulated communication link using CST

Fig. 6 Communication link

\subsection{Transmitter and receiver carrying OAM mode number of $l=0$}

In this part, elements of the receiver and the transmitter in the communication link in the same phase of $\varphi=0$, which are providing OAM mode number of $l=0$.

In Fig. 7, schematic of a two-port network has been presented. Elements 1 to 12 constitute the elements of the transmitter and elements 13 to 24 constitute the elements of the receiver. Each element has a controlled phase shifting feeding network. For such two-port network, $S_{21}$ parameter is defined as follows.

$$
\left|S_{21}\right|=10 \log \frac{P_{2}}{P_{1}}
$$
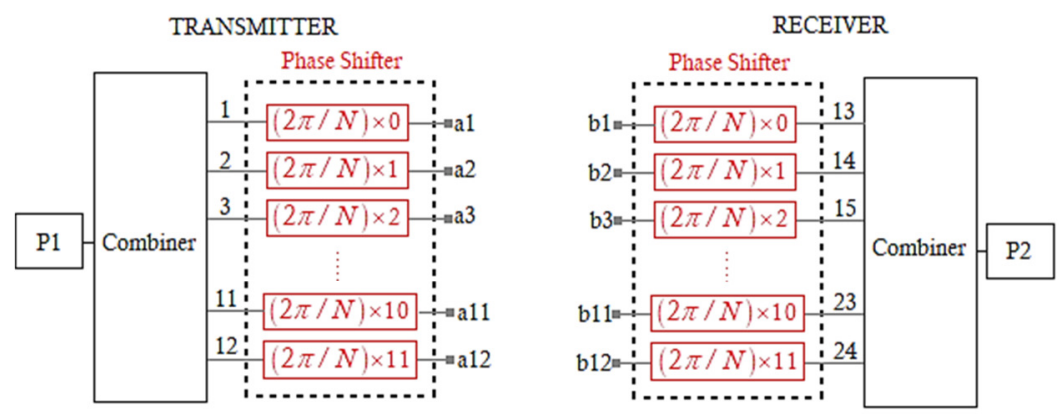

Fig. 7 Schematic of the two-port network

In the proposed communication link, the receiver is located at the distances of $1000(8 \lambda) m m, 2000(16 \lambda) m m$ and $5000(40 \lambda) \mathrm{mm}$ away from the transmitter. The diagram of the insertion loss between the ports of the transmitter and the receiver, calculated as $S_{21}$ parameter for different distances is presented in Fig. 8.

The obtained values of $S_{21}$ are $-16.9 \mathrm{~dB},-21.6 \mathrm{~dB}$ and $-31.4 \mathrm{~dB}$ for the distances of $1000 \mathrm{~mm}, 2000 \mathrm{~mm}$ and $5000 \mathrm{~mm}$, respectively. 
According to Fig. 8, as expected, increasing the distance of the receiver from the transmitter causes a decrease in the level of the receiving signal from the aperture of the receiver.

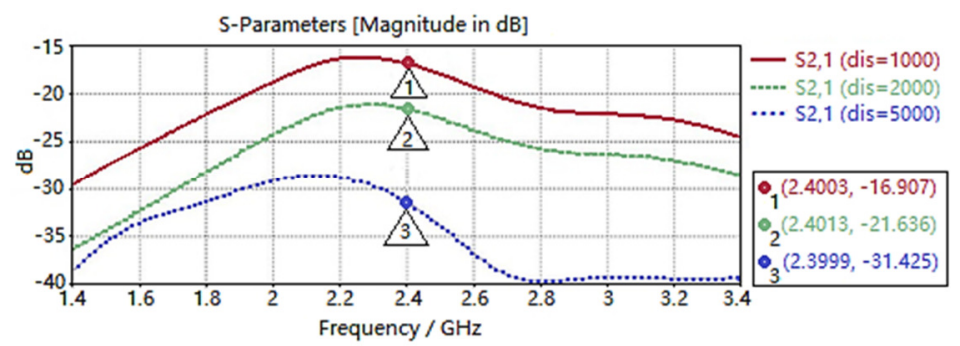

Fig. 8 Diagram of the insertion loss

\subsection{Transmitter carrying OAM mode number of $l=1$ and receiver carrying OAM mode number of $l=0$}

In this part, a circular phased array antenna with a feeding network generating OAM mode number of $l=1$ is designed as a transmitter, while the elements of the receiving antenna have the same phase, supporting OAM mode number of $l=0$. The diagram of the insertion loss between the ports of the receiver and the transmitter calculated as $S_{21}$ parameter for different distances is presented in Fig. 9.

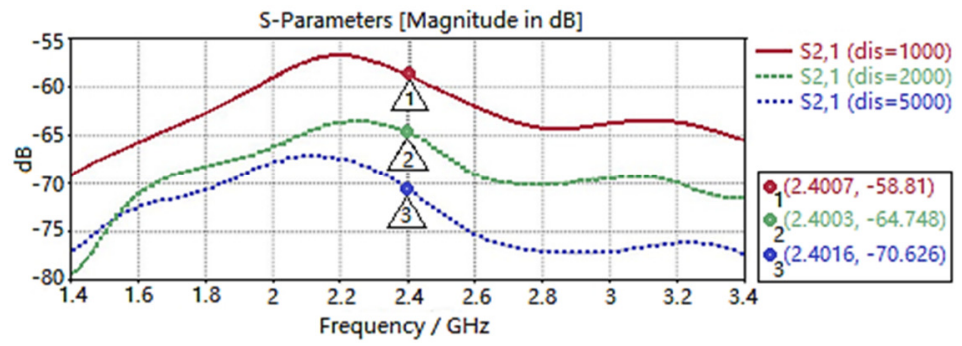

Fig. 9 Diagram of the insertion loss

The obtained values of $S_{21}$ are $-58.8 \mathrm{~dB},-64.7 \mathrm{~dB}$ and $-70.6 \mathrm{~dB}$ for the distances of, $1000 \mathrm{~mm}, 2000 \mathrm{~mm}$, and $5000 \mathrm{~mm}$, respectively.

Comparison of Fig. 8 with Fig. 9 shows that when OAM mode numbers in the receiver and the transmitter are different, the insertion loss in the proposed link is increased significantly. This shows low interference between the end ports of the transmitter and the receiver. Fig. 9 also shows that increasing the distance between the receiver and the transmitter causes the drop of receiving signal level, as expected.

\subsection{Transmitter and receiver carrying OAM mode number of $l=1$}

In this part, the transmitter and the receiver are circular array antennas carrying OAM mode number of $l=1$. The diagram of the insertion loss between the ports of the receiver and the transmitter calculated as $S_{21}$ parameter for different distances is presented in Fig. 10.

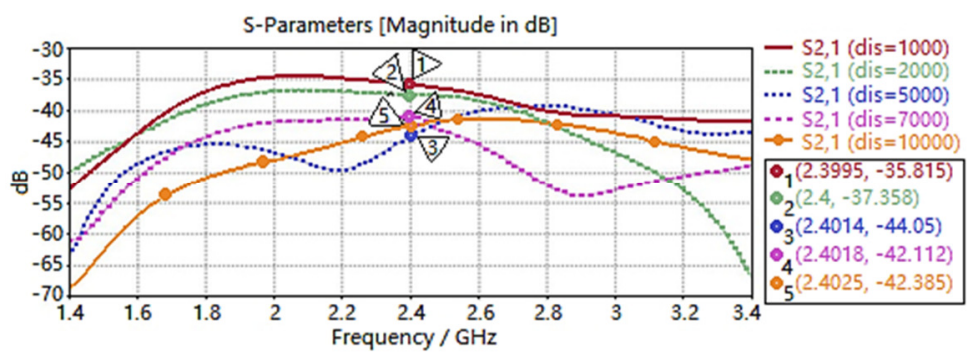

Fig. 10 Diagram of the insertion loss

The obtained values of $S_{21}$ are $-35.8 d B,-37.4 d B,-44.1 d B,-42.1 d B$ and $-42.4 d B$ for the distances of $1000 \mathrm{~mm}, 2000 \mathrm{~mm}$, $5000 \mathrm{~mm}, 7000 \mathrm{~mm}$, and $10000 \mathrm{~mm}$, respectively. 
By comparing Fig. 8 and Fig. 10 with Fig. 9, it is observed that when the transmitter and the receiver have the same OAM mode numbers, the received signal level is relatively higher than when the modes are different. Therefore, the full wave simulation shows that the receiver in an OAM link receives the same mode number from the transmitter rather than other mode numbers demonstrating the orthogonality between them. This also indicates low interference between different OAM modes. By comparing Fig. 8 with Fig. 10, it can be observed that increasing the mode number of OAM antennas decreases the level of the receiving signal as expected due to the presence of a null at their boresight direction.

\section{Definition of Effective OAM Gain}

\subsection{Defining a reference gain}

Assume that we have an OAM link with two circular array antennas in front of each other. Due to the presence of the boresight null in the radiation pattern of OAM antennas, the observed gain on the axis of the link where the null is observed is negligible, theoretically zero. Therefore, assuming $\theta=0$ to define the effective gain in an OAM link will result in a very low effective gain, which is of course an undesired point for OAM links. To resolve such a problem, one can assume that two OAM antennas in a communication link are visible by each other in angles up to $\theta=\tan ^{-1}(R / d)$ above and less than $\theta=0$, according to Fig. 6(a). By assuming angle $\theta$ as the higher angle from which the other antenna can be observed, we can define non-zero OAM gain in the direction of the other antenna as a reference gain, which can be later used. It is clear that we don't expect this value can be used in Friis Transmission Formula.

\subsection{Using Friis formula}

When there is an antenna link, we are able to calculate the effective gain of each antenna by using Friis Transmission Equation. In [21-22], Friis Transmission Formula for an OAM link with two circular phased array antennas was used and discussed to evaluate the gain of the link. It is shown by them that the Friis formula should be manipulated in order to fit OAM links by introducing a new formulation which depends on $1 / d^{(2+2|l|)}$ instead of $1 / d^{2}$. The obtained result was dependent on variables such as the number of the elements of the circular arrays, the radius of the arrays, OAM mode number of the antennas and also the distance of the receiver from the transmitter.

However, having the well-known Friis Transmission Formula as a basic equation to calculate the link budget, we use the same simple formula to demonstrate the superiority of OAM links over none-OAM cases. Assume that a receiver and a transmitter with the gain of $G_{R}$ and $G_{T}$, and the power of $P_{R}$ and $P_{T}$, respectively, are placed in a communication link, separated by a distance of $d$ and operating at the frequency of $f$, if $P_{T}$ watts of total power from the source are delivered to the transmitter, the received power in the receiver, can be written as follows [4].

$$
P_{R}=\frac{P_{T} G_{T}(\theta, \varphi) G_{R}(\theta, \varphi) \lambda^{2}}{(4 \pi d)^{2}}=\frac{P_{T} G_{e f f}^{2} \lambda^{2}}{(4 \pi d)^{2}}
$$

Eq. (8) relates the free space path loss, antenna gains and wavelength, with the power of the receiver and the transmitter antennas. When the receiving and the transmitting antennas are exactly the same, the relation becomes simpler by having only one gain parameter. By having full wave results of a link simulation from the previous section and also using (7), we are able to calculate antenna's effective gain $G_{\text {eff }}$ from the $S_{21}$ results of the link in a simpler form as shown in (9).

$$
\left|S_{21}\right|^{2}=\frac{G_{e f f}^{2} \lambda^{2}}{(4 \pi d)^{2}}
$$


Friis Transmission Equation is valid when the antennas are in each other's far field region. Therefore, the far field of the proposed circular array antenna is calculated as $\left(2 D^{2}\right) / \lambda=1000 \mathrm{~mm}$. Thus, when the receiver is placed farther than the distance of $d=1000 \mathrm{~mm}$ from the transmitter, Friis Transmission Equation can be applied with acceptable accuracy.

To calculate the effective gain of an antenna in an OAM link with antennas carrying OAM mode number of $l=0$, Friis Transmission Equation has been considered for different distances of the receiver from the transmitter which are in the far field of the transmitting antenna. According to (9), for $l=0$, the effective gain is calculated by using Fig. 8 and is presented in Table 1 .

Table 1 Results of the communication link in Fig. 8

\begin{tabular}{|c|c|c|}
\hline$d(m m)$ & $S_{21}(d B)$ & $G_{\text {eff }}(d B)$ \\
\hline 2000 & -22 & 12.03 \\
\hline 5000 & -31 & 11.5 \\
\hline
\end{tabular}

The obtained values of the effective gain are $12.03 \mathrm{~dB}$ and $11.5 \mathrm{~dB}$ for the distances of $2000 \mathrm{~mm}$ and $5000 \mathrm{~mm}$ respectively, which are equal to the simulation results of the radiation pattern in Fig. 4 . This validates the results of this method with the accuracy of around $1 d B$.

\subsection{Defining OAM gain}

Then, proceeding to OAM mode number of $l=1$, the effective gain in the communication link with antennas carrying OAM mode number of $l=1$ is calculated according to the obtained values of $S_{21}$ in Fig. 10 and presented in Table 2 . The obtained results are $4.3 \mathrm{~dB}, 5.01 \mathrm{~dB}, 7.4 \mathrm{~dB}$ and $8.8 \mathrm{~dB}$ for the distances of $2000 \mathrm{~mm}, 5000 \mathrm{~mm}, 7000 \mathrm{~mm}$ and $10000 \mathrm{~mm}$, respectively.

In order to have a fair comparison, the calculated effective gain is compared with the observed gain by one of the antennas view angles from the other one, namely as shown in Fig. 1. The 2D radiation pattern of the antenna carrying OAM mode number of $l=1$ has presented in Fig. 11. The view angle of each antenna by the other one for different distances has been calculated using (10).

$$
\theta=\tan ^{-1}\left(\frac{R}{d}\right)
$$
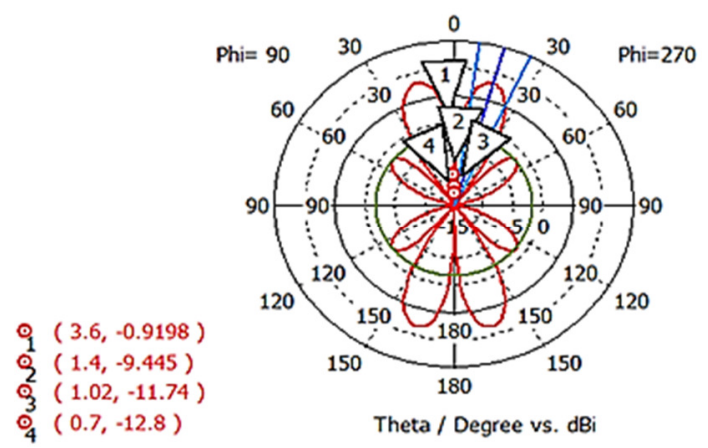

Fig. $112 \mathrm{D}$ radiation pattern of the circular array antenna

As shown in Fig. 11, the obtained values of $G_{\theta}$ are $-0.92 d B,-9.45 d B,-11.74 d B$ and $-12.8 d B$ for the distances of $2000 \mathrm{~mm}, 5000 \mathrm{~mm}, 7000 \mathrm{~mm}$, and $10000 \mathrm{~mm}$, respectively. Thus, we are able to define a useful parameter called OAM Gain as the difference between the two as follows.

$$
O A M_{G a i n} d B=G_{e f f} d B-G_{\theta} d B
$$

This parameter indicates that how much an OAM system improves the obtained signal level at the receiver side compared to the maximum gain value, which is obtained from its radiation pattern in the broadside direction of the other antenna. The 
parameter can simplify the calculation of the link budget in such cases. Table 2 shows the results of the $S_{21}$ parameter, the calculated effective gain $\left(G_{e f f}\right)$, the effective area of the aperture $\left(A_{e f f}\right)$, the view angle of each antenna by the other one $(\theta)$ and its related gain $\left(G_{\theta}\right)$, and the OAM gain $\left(O A M_{\text {Gain }}\right)$ of the communication link presented in Fig. 10 for different distances. The view angle of each antenna by the other one is determined in Fig. 6(a), and the effective area of the aperture is calculated using the following equation

$$
A_{e f f}=\frac{\lambda^{2}}{4 \pi} G_{e f f}
$$

Table 2 Results of the communication link in Fig. 10

\begin{tabular}{|c|c|c|c|c|c|c|}
\hline $\mathrm{d}(\mathrm{mm})$ & $\mathrm{S}_{21}(\mathrm{~dB})$ & $\mathrm{G}_{\text {eff }}(\mathrm{dB})$ & $\mathrm{A}_{\text {eff }}\left(\mathrm{m}^{2}\right)$ & $\Theta\left(^{\circ}\right)$ & $\mathrm{G}_{\theta}(\mathrm{dB})$ & $\mathrm{OAM}_{\text {Gain }}(\mathrm{dB})$ \\
\hline 2000 & -37.4 & 4.3 & 0.0028 & 3.6 & -0.92 & 5.22 \\
\hline 5000 & -44.1 & 5.01 & 0.0031 & 1.4 & -9.45 & 14.46 \\
\hline 7000 & -42.1 & 7.4 & 0.0055 & 1.02 & -11.74 & 19.14 \\
\hline 10000 & -42.4 & 8.8 & 0.0079 & 0.7 & -12.8 & 21.6 \\
\hline
\end{tabular}

In order to show the advantage of OAM link, two simulations by using a communication link with a transmitter carrying OAM mode number of $l=1$ to a receiver carrying OAM mode number of $l=0$ are performed. The effective gain for the two other combinations has been calculated using Fig. 9 and have been presented in Table 3 . The obtained values are $-9.5 d B$ and $-8.5 \mathrm{~dB}$ for the distances of $2000 \mathrm{~mm}$ and $5000 \mathrm{~mm}$, respectively. This makes us claim that an antenna with OAM mode number of $l$ receives the information of a transmitter with the same OAM mode number, which makes the communication link secure.

Table 3 Results of the communication link in Fig. 9

\begin{tabular}{|c|c|c|}
\hline$d(m m)$ & $S_{21}(d B)$ & $G_{\text {eff }}(d B)$ \\
\hline 2000 & -65 & -9.5 \\
\hline 5000 & -71 & -8.5 \\
\hline
\end{tabular}

\section{Conclusion}

With reference to the nature of electromagnetic waves, orthogonality of different modes makes it possible to transmit several categories of information simultaneously at a single frequency. In the presented paper, this property of electromagnetic waves has been analyzed using orbital angular momentum (OAM). For this purpose, we have simulated a circular phased array antenna generating beams carrying OAM and then used them as a transmitter and a receiver in a communication link. For non-zero OAM links, the gain parameter cannot be calculated as usual because of the presence of a null in the beam axis of the radiation pattern of such antennas. By using two new gain parameters, we defined the concept of OAM gain for the first time in order to evaluate OAM links. Nonzero OAM beams can be used in communication links because of their unlimited number of modes. A desirable point of OAM antennas may be achieved by decreasing the depth and the width of the created null.

\section{Conflicts of Interest}

The authors declare no conflict of interest.

\section{References}

[1] Y. Yan, G. Xie, M. P. Lavery, H. Huang, N. Ahmed, C. Bao, et al., "High-Capacity Millimetre-Wave Communications With Orbital Angular Momentum Multiplexing,” Nature Communications, vol. 5, no. 1, pp. 1-9, September 2014.

[2] N. Bozinovic, Y. Yue, Y. Ren, M. Tur, P. Kristensen, H. Huang, et al., "Terabit-Scale Orbital Angular Momentum Mode Division Multiplexing in Fibers," Science, vol. 340, no. 6140, pp. 1545-1548, June 2013. 
[3] F. Tamburini, E. Mari, A. Sponselli, B. Thidé, A. Bianchini, and F. Romanato, "Encoding Many Channels on the Same Frequency through Radio Vorticity: First Experimental Test," New Journal of Physics, vol. 14, no. 3, 033001, March 2012.

[4] O. Edfors and A. J. Johansson, "Is Orbital Angular Momentum (OAM) Based Radio Communication an Unexploited Area?" IEEE Transactions on Antennas and Propagation, vol. 60, no. 2, pp. 1126-1131, February 2012.

[5] M. Zyczkowski, K. D. Brewczynski, and M. Karol, "Innovative Security Technology for Optical Fiber Data Transmission Using Optical Vortex,” Proceedings of Engineering and Technology Innovation, vol. 5, no. 1, pp. 1-6, August 2017.

[6] L. Allen, M. W. Beijersbergen, R. J. C. Spreeuw, and J. P. Woerdman, "Orbital Angular Momentum of Light and the Transformation of Laguerre-Gaussian Laser Modes,” Physical Review A, vol. 45, no. 11, pp. 8185-8189, June 1992.

[7] M. W. Beijersbergen, R. P. C. Coerwinkel, M. Kristensen, and J. P. Woerdman, "Helical-Wavefront Laser Beams Produced with a Spiral Phaseplate,” Optics Communications, vol. 112, no. 5-6, pp. 321-327, December 1994.

[8] S. M. Mohammadi, L. K. Daldorff, J. E. Bergman, R. L. Karlsson, B. Thidé, K. Forozesh, et al., "Orbital Angular Momentum in Radio-A System Study," IEEE Transactions on Antennas and Propagation, vol. 58, no. 2, pp. 565-572, February 2010.

[9] M. Veysi, C. Guclu, and F. Capolino, "Vortex Beams with Strong Longitudinally Polarized Magnetic Field and their Generation by Using Metasurfaces," Journal of the Optical Society of America B, vol. 32, no. 2, pp. 345-354, February 2015.

[10] M. S. Soskin, V. N. Gorshkov, M. V. Vasnetsov, J. T. Malos, and N. R. Heckenberg, “Topological Charge and Angular Momentum of Light Beams Carrying Optical Vortices,” Physical Review A, vol. 56, no. 5, pp. 4064-4075, November 1997.

[11] J. Wang, J. Y. Yang, I. M. Fazal, N. Ahmed, Y. Yan, H. Huang, et al., “Terabit Free-Space Data Transmission Employing Orbital Angular Momentum Multiplexing," Nature Photonics, vol. 6, no. 7, pp. 488-496, July 2012.

[12] B. Thidé, H. Then, J. Sjöholm, K. Palmer, J. Bergman, T. D. Carozzi, et al., "Utilization of Photon Orbital Angular Momentum in the Low-Frequency Radio Domain," Physical Review Letters, vol. 99, no. 8, 087701, August 2007.

[13] D. Zelenchuk and V. Fusco, "Split-Ring FSS Spiral Phase Plate," IEEE Antennas and Wireless Propagation Letters, vol. 12, pp. 284-287, 2013.

[14] X. Hui, S. Zheng, Y. Chen, Y. Hu, X. Jin, H. Chi, et al., "Multiplexed Millimeter Wave Communication with Dual Orbital Angular Momentum (OAM) Mode Antennas,” Scientific Reports, vol. 5, no. 1, 10148, May 2015.

[15] A. M. Yao and M. J. Padgett, “Orbital Angular Momentum: Origins, Behavior and Applications," Advances in Optics and Photonics, vol. 3, no. 2, pp. 161-204, June 2011.

[16] J. D. Jackson, “Classical Electrodynamics, 3rd ed.” American Journal of Physics, vol. 67, no. 9, 841, September 1999.

[17] L. Josefsson and P. Persson, Conformal Array Antenna Theory and Design, 1st ed. Hoboken: John Wiley \& Sons, Inc., 2006.

[18] T. B. Vu, "Side-Lobe Control in Circular Ring Array," IEEE Transactions on Antennas and Propagation, vol. 41, no. 8, pp. 1143-1145, August 1993.

[19] C. A. Balanis, Antenna Theory: Analysis and Design, 4th ed. Hoboken: John Wiley \& Sons, Inc., 2016.

[20] A. F. Morabito, L. Di Donato, and T. Isernia, "Orbital Angular Momentum Antennas: Understanding Actual Possibilities through the Aperture Antennas Theory,” IEEE Antennas and Propagation Magazine, vol. 60, no. 2, pp. 59-67, April 2018.

[21] D. K. Nguyen, O. Pascal, J. Sokoloff, A. Chabory, B. Palacin, and N. Capet, "Discussion about the Link Budget for Electromagnetic Wave with Orbital Angular Momentum," The 8th European Conference on Antennas and Propagation, April 2014, pp. 1117-1121.

[22] D. K. Nguyen, O. Pascal, J. Sokoloff, A. Chabory, B. Palacin, and N. Capet, "Antenna Gain and Link Budget for Waves Carrying Orbital Angular Momentum,” Radio Science, vol. 50, no. 11, pp. 1165-1175, November 2015.

Copyright $(\mathrm{C}$ by the authors. Licensee TAETI, Taiwan. This article is an open access article distributed under the terms and conditions of the Creative Commons Attribution (CC BY-NC) license (https://creativecommons.org/licenses/by-nc/4.0/). 